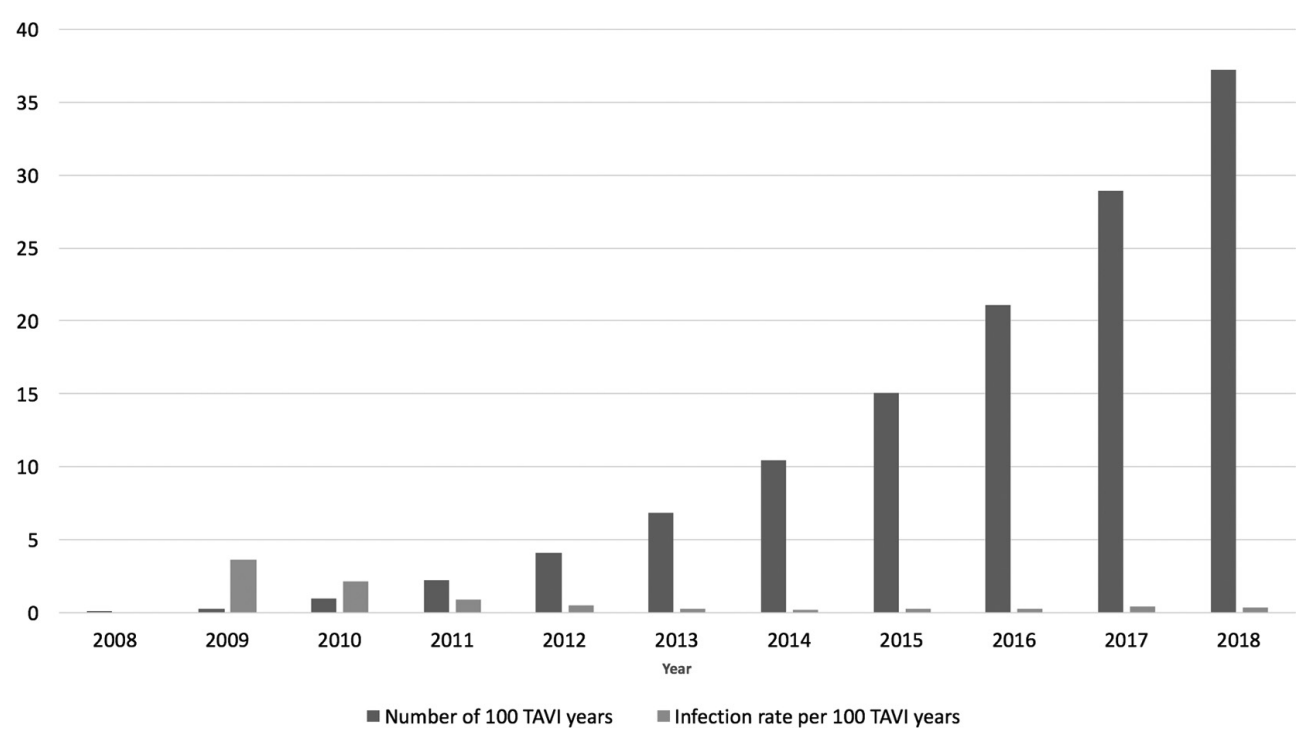

Abstract 129 Figure 2 PVE rate per 100 TAVI years relative to cumulative 100 TAVI years

of TAVI-PVE, due to lack of diagnostic echocardiographic features. This may be a result of TAVI-PVE being less likely to demonstrate new paravalvular leak than SAVR-PVE due to the absence of suturing material in the former. As such, a high index of suspicion is required.

Conflict of Interest None

\section{FEVER IN AN INTRAVENOUS DRUG USER: IF IT ISN'T ENDOCARDITIS THEN WHAT IS IT?}

${ }^{1}$ Sam Straw*, 'Wazir Baig, ${ }^{2} J i a n h u a$ Wu, ${ }^{1} J o n a t h a n$ Sandoe. ' Leeds Teaching Hospitals NHS Trust; ${ }^{2}$ University of Leeds

10.1136/heartjnl-2019-BCS.127
Background Intravenous drug use (IVDU) is a predisposition for the development of infective endocarditis (IE). The incidence amongst people who inject drugs (PWID) is estimated to be 50-100 times greater than the general population. For this reason, PWID with symptoms of infection or bacteraemia are frequently referred to the IE team for assessment. We have previously reported on our experience of managing PWID with IE over more than a decade. Here we present the characteristics, final diagnoses and outcomes in all PWID admitted to hospital with other infections in whom IE was suspected.

Methods Patients aged 18 or over who had taken drugs intravenously within 90 days and referred to the IE team between 01/01/2006 and 31/12/2016 were eligible. Inclusion was dependent on the modified Duke criteria, we required

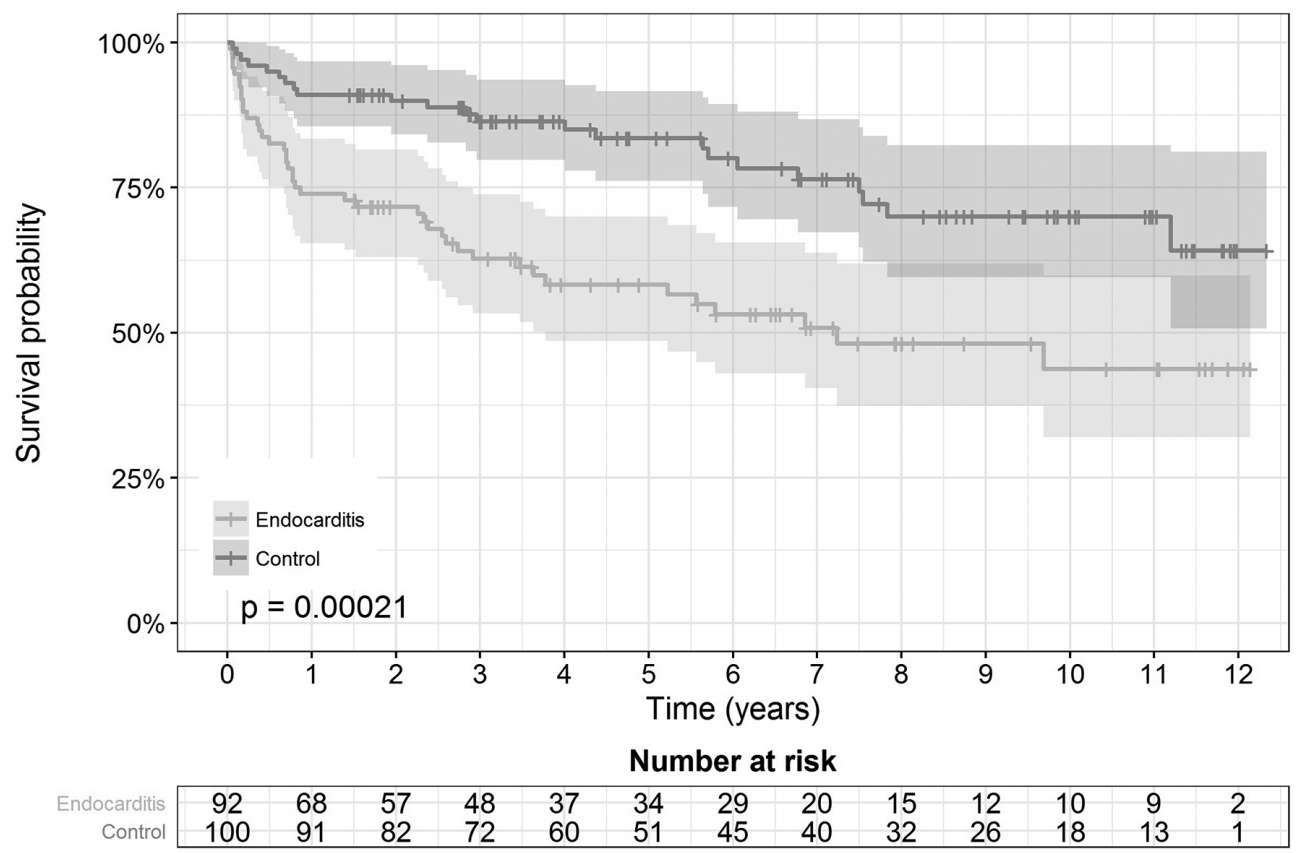

Abstract 130 Figure 1 